\title{
Phonological similarity effects on detecting change in simple arrays
}

\author{
STEPHEN MONDY and VERONIKA COLTHEART \\ Macquarie University, Sydney, New South Wales, Australia
}

\begin{abstract}
In two experiments, we investigated the effects on change detection and identification of the phonological similarity of the names of drawn objects shown in simple arrays. Experiment 1 examined the effects of phonological similarity on report of identity changes (in which one object was replaced by another) in a five-item array and Experiment 2 its effects on reporting switch changes (in which objects exchanged places). Phonological similarity did not impair change detection, and identification of new objects on change trials was more accurate in Experiment 1. However, phonological similarity significantly reduced change detection and identification of objects that switched location in Experiment 2. The results indicate different roles for a visual short-term memory system such as the visuospatial sketchpad and for phonological coding and storage in detecting and identifying replaced objects and object locations in arrays.
\end{abstract}

Subjects in change-detection experiments fail to detect large changes made to scenes or arrays of objects when those changes are made during saccades (Grimes, 1996; McConkie \& Currie, 1996) or during simulated saccades such as occlusions. This change blindness has been demonstrated for occlusions and partial occlusions of static scene displays (O’Regan, Rensink, \& Clark, 1996; Simons, 1996), in scenes viewed under "flicker" conditions (Rensink, O'Regan, \& Clark, 1995), in dynamic scenes such as film and video clips (Levin \& Simons, 1997), in virtual reality environments (Wallis \& Bülthoff, 2000), and in real-world interactions (Simons \& Levin, 1998). Attention has been highlighted as an important factor in detecting change under such conditions (Rensink et al., 1995). Typically, if subjects fail to direct attention to items in scenes (for example, to items that may be of marginal interest), then they fail to detect changes involving those items.

The change-detection paradigm has been used to investigate the nature of visual memory. Simons (1996), for example, presented subjects with a display on a computer screen for $2 \mathrm{sec}$, followed by an interstimulus interval of $4.3 \mathrm{sec}$, and then a second display that was either identical to the first, or one in which a change had been made to a single element of the display. Each display consisted of an array of five objects arranged in a $3 \times 3$ matrix to which three types of changes were made. First, an object could

This research was supported by ARC Special Research Centre Scheme (2000-2008) to the Macquarie Centre for Cognitive Science. Correspondence concerning this article should be addressed to V. Coltheart, Macquarie Centre for Cognitive Science, Macquarie University, Sydney, NSW 2109, Australia (e-mail: veronika@maccs.mq.edu.au).

Note-This article was accepted by the previous editorial team, when Colin M. MacLeod was Editor. undergo an identity change (be replaced at the same array location by an object not in the original array; the identity condition). Second, two objects could exchange locations with each other (the switch condition). Third, a single object could "move" from its original position in the matrix to a previously unoccupied cell (the configuration condition). In this condition, a previously occupied cell became empty and a formerly empty, blank area was now filled by an object. The subjects' task was to decide if the second array was identical to, or different from, the first array (chance performance was therefore 50\%). The pattern of results in Simons's (1996) first experiment showed that change detection was best for configuration changes (nearly $100 \%$ ) and significantly lower for the identity and switch conditions (which did not differ significantly from each other, although both differed significantly from chance).

Because all of the subjects reported using a covert naming strategy in that experiment (which may have improved accuracy in both the identity and switch conditions), three further experiments used novel (hence, difficult-to-name) black geometric shapes as stimuli. The first of these replicated the results of the real-object experiment just described, with accurate detection of configuration changes, but reduced accuracy in detecting identity and switch changes relative to performance with nameable objects. The second and third of these further experiments using difficult-to-name stimuli manipulated the frequency of stimulus repetition and the duration of the interstimulus interval, but found a broadly similar pattern of performance as did the earlier experiments. In summary, these experiments found that as naming difficulty increased, as a result of experimental manipulation, detection of change declined. The last of Simons's (1996) experiments used photographs of nameable objects, but with an additional interference task designed to reduce verbal labeling: Subjects concurrently shadowed a recording of a story while 
viewing the arrays. This concurrent articulation significantly reduced detection in both the identity and switch conditions (to chance, in the case of the switch condition) in comparison with performance in both conditions of his Experiment 1, when concurrent articulation was not required.

The results of these experiments confirmed the findings of others that subjects tend to use verbal encoding strategies spontaneously when faced with simple short-term memory (STM) tasks (Avons \& Phillips, 1987; Conrad, 1964; Noizet \& Pynte, 1976; Zelinsky \& Murphy, 2000). Zelinsky and Murphy, for example, found that subjects employed an implicit naming strategy when encoding objects for later recognition, and that this acted as an oculomotor constraint on visual inspection: Pictures with longer names were fixated longer than pictures with shorter names. They concluded that faster visual processing was constrained to wait until slower linguistic processes were completed.

Simons's (1996) results further showed that detection of global configuration changes was not impaired either by reducing the nameability of the stimuli or by verbal interference. As Simons noted, the global spatial layout of an array may be directly visually encoded, using an independent system that does not interact with naming processes. Such an independent system might be the visuospatial sketchpad of Baddeley's (1986) model of short-term working memory. In this well-known model, the memory system consists of a number of components: a supervisory central executive and two slave systems (the phonological loop and the visuospatial sketchpad). The phonological loop processes and maintains verbally coded material and the visuospatial sketchpad maintains visual information and spatially coded material. The two systems function independently, so configuration changes (along with other forms of visual information) may be directly encoded by the visuospatial sketchpad.

Simple arrays of objects (and objects embedded in more naturalistic scenes) differ, as stimuli, from either lists of pictures or lists of words, because in typical working memory tasks, the objects or words in such lists are usually presented serially in the same spatial location. The objects in arrays and scenes, by contrast, are spatially dispersed and thus have a configural or contextual component (where the context may just be the other objects in the array). For example, in Simons's (1996) configuration change condition, an object "moved" to an unoccupied cell, creating changes to the overall or global "shape" of the array. Such visual relational information in a simple display appears to be obligatorily encoded (Jiang, Chun, \& Olson, 2004; Jiang, Olson, \& Chun, 2000; Woodman, Vecera, \& Luck, 2003). Identification and comprehension of objects in a scene are not dependent on covert naming (which is substantially slower than reading object names aloud, as shown by Potter \& Faulconer, 1975), because people appear to comprehend at least the gist of briefly presented scenes when these are shown at rates of about $10 / \mathrm{sec}$, under conditions unlikely to permit name retrieval.
In both Simons's (1996) identity and switch manipulations, verbal interference significantly reduced change detection, leading to his suggestion that in the absence of verbal encoding, "no visual information about object properties is preserved across views of a scene" (p. 304). Such a strong statement cannot account for all visual memory for scenes, and has since been qualified by Simons and Rensink (2005), but verbal encoding, retrieval of object names, and rehearsal of the names all appear to play an important part in the retention of information in arrays and scenes. The concurrent shadowing task used by Simons (1996) not only reduced the likelihood of verbal encoding of scene objects but also divided attention and, as noted by Rensink et al. (1995), attention to objects prior to change is a necessary, if not sufficient, condition for successful change detection to occur. Consequently, it is possible that other manipulations that are known to reduce the ability of observers to convert visual to phonological codes will in turn reduce the accuracy of change detection.

Phonological similarity of items in word or letter lists, for example, reduces accurate serial recall and does not impose the dual-task demands of concurrent articulation (Baddeley, 1966, 1968, 1986; Coltheart, 1993; Conrad, 1963, 1964; Conrad \& Hull, 1964; Hintzman, 1965, 1967; Longoni, Richardson, \& Aiello, 1993; Schweickert, Guentert, \& Hersberger, 1990; Wickelgren, 1965). In accounts of working memory, it was proposed that visual stimuli undergo phonological recoding and that the phonological codes are registered in a phonological short-term store, refreshed and maintained by subvocal rehearsal. The store and the rehearsal process together constitute the phonological loop (Baddeley, 1986). Words (or the names of pictures) that are phonologically similar have many overlapping features, and this may result in item "confusions" during maintenance in the system (Baddeley, 1968, 1986), or subsequent errors at retrieval (Nairne, 1990).

Phonological similarity effects are not limited to word lists, however. Phonological similarity of picture names disrupts recall of lists of pictures in older children and adults (Coltheart, 1999; Conrad, 1971, 1972; Schiano \& Watkins, 1981). Children under 5 do not rely on speechbased codes in memory for drawings (Halliday, Hitch, Lennon, \& Pettipher, 1990; Hitch \& Halliday, 1983; Hitch, Woodin, \& Baker, 1989; Longoni \& Scalisi, 1994) and do not show phonological similarity effects until phonological recoding of visual stimuli begins to generalize across memory tasks, as children become familiar with reading (Longoni \& Scalisi, 1994).

Phonological similarity of items - or their names, in the case of pictures - does not usually abolish all recall; instead, it results in substantially lower levels of performance. Similarly, concurrent articulation reduces recall, and removes phonological similarity and word-length effects on recall, but a reduced number of items are nevertheless retained. Recall under these conditions may be (1) mediated by a residual, limited degree of phonological encoding and reduced levels of rehearsal, (2) due to 
reliance on other forms of encoding, such as visual or semantic codes that also contribute to working memory retention, or (3) due to both (Avons \& Phillips, 1987; Poirier \& Saint-Aubin, 1996).

When people were shown lists of four or five pictures that had either phonologically similar or dissimilar names, at rates of 1/sec (Coltheart, 1999; Schiano \& Watkins, 1981 ) and $8 / \sec$ (Coltheart, 1999), phonological similarity reduced recall at slow $(1 / \mathrm{sec})$ rates under both serial and free recall scoring, and concurrent articulation (coincident irrelevant speech) abolished the effect. Although phonological similarity effects were not found at high rates of presentation (8/sec; Coltheart, 1999), it is likely that during normal visual inspection of scenes, given fixation durations of up to $500 \mathrm{msec}$, phonological codes have sufficient time to be activated and registered in the phonological short-term store of working memory. It is not yet known whether phonological similarity of the names of objects in visual scenes has any effect on the ability of observers to report changes in those scenes. When arrays of four or six consonant letters were shown for $250 \mathrm{msec}$, followed by a probe letter after $3 \mathrm{sec}$, MacAndrew, Klatzky, Fiez, McClelland, and Becker (2002) found no adverse effects of phonological similarity on detection of probes from the preceding array. They concluded that this task can be performed without reliance on phonological codes. However, the brief presentation may have limited the possible use of phonological encoding of letter names; in changedetection experiments, in most paradigms, the stimuli are available for several seconds.

When the task is to study and remember a visual stimulus for a few seconds in order to be able to detect and report any change occurring in a subsequent stimulus, we assume that systems such as the visuospatial sketchpad, as well as active verbal encoding by means of the phonological loop, will be invoked and engaged in establishing representations of the information in the pair of stimuli. The central executive controls the allocation of attention and the application of specific encoding strategies, and engages in comparison and decision processes. A mismatch of information in the second stimulus and the information stored by the visuospatial sketchpad might provide input to the central executive, provoking the decision that something has changed, but the change might not be explicitly describable. Using repeatedly alternating stimuli in the flicker task, Rensink (2004) observed a delay between detection of change and the ability to report the nature of the change. Of course, there is likely to be a continuum in the extent to which the specific details of a change can be described. The possible contribution of the phonological loop can be examined through the use of two dependent variables: change detection and identification of changed items. It is possible that change detection can be based on information stored in the visuospatial sketchpad so that phonological similarity of item names will not affect performance. If phonological coding contributes to this task, then phonological similarity can be expected to impair performance. Change identification does require the phonological loop, because the changes must be verbally reported, and phonological similarity could affect response accuracy.

The experiments reported here thus examined whether change detection was subject to the effects of phonological similarity. Observers were shown an array of five line drawings of common objects, which was briefly masked. The postmask array either remained unchanged, or one of the items in the array was replaced by a new item (Experiment 1), or two objects exchanged locations (Experiment 2). Phonological similarity of the names of the arrayed objects was manipulated so that in half the trials, the names of the objects in the arrays were phonologically similar, and in half they were phonologically dissimilar. In each case, the new object had a name that was phonologically similar to the object it replaced. It was anticipated that, if observers covertly name and attempt to maintain phonological codes representing object names between the first and second displays, phonological similarity of the object names would reduce their ability to retain the names for comparison with the objects present in the second display. Of course, as noted earlier, the comparison is likely to depend on stored visual information as well as on memory for phonological name codes.

\section{EXPERIMENT 1}

Experiment 1 examined the effects on change detection and identification of the phonological similarity of the names of objects present in an array. Five objects were displayed in an imaginary nine-cell matrix in a task similar to that of Simons (1996). The phonologically similar sets of objects shared their first consonant phoneme and, in many cases, their vowel phoneme. This form of similarity has led to inferior recall in STM tasks (Baddeley, 1966), whereas shared rhymes (vowel and final consonant overlap) sometimes produce enhanced recall when contrasted with recall of dissimilar words (Fallon, Groves, \& Tehan, 1999). For dissimilar arrays, the object names shared few phonemes except that the replaced object on a change trial had a name phonologically similar to the replaced object's name.

\section{Method}

Subjects. A sample of 26 (21 female) Macquarie undergraduates (mean age 20.8 years) participated for course credit. All reported normal or corrected-to-normal vision.

Apparatus. Stimuli were displayed on a white background on a 17-in. Apple Macintosh eMac monitor (screen resolution 1,024 $\times$ 768 pixels) controlled by an $800-\mathrm{MHz}$ G4 personal computer running PsyScript software (Bates \& D’Oliveiro, 2003).

Stimuli. The stimuli were line-drawn pictures from a subset of the Snodgrass and Vanderwart (1980) corpus (plus some drawn by the first author) and included inanimate objects, living things, and body parts. (Although all were not inanimate and all not whole objects, they are referred to as "objects" here, in the interest of brevity.) On each trial, five objects were assigned to one of 9 positions in an imaginary $3 \times 3$ array, centered on the screen. The position of each object in the array was randomly determined, with the caveat that, across all trials, the "change" object appeared an approximately equal number of times at each array position. See Figure 1 for examples of similar and dissimilar arrays. 
Similar Trial

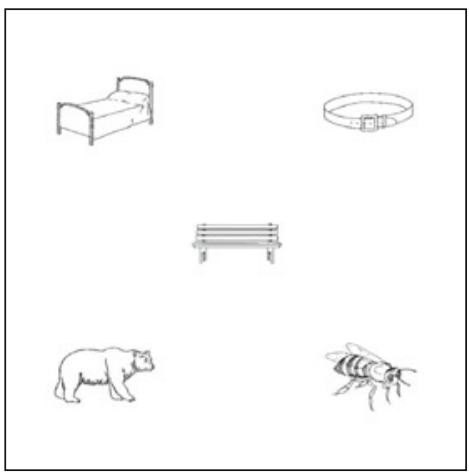

Array 1

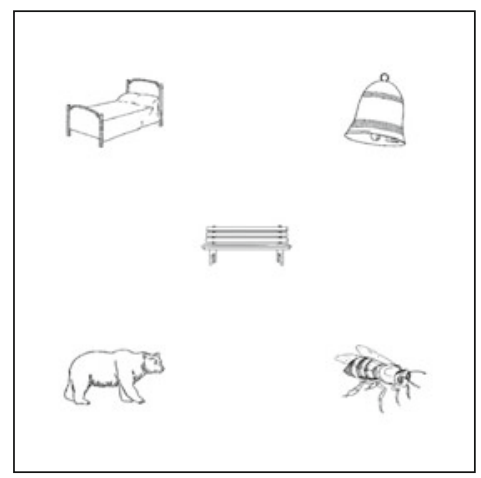

Array 2

Dissimilar Trial

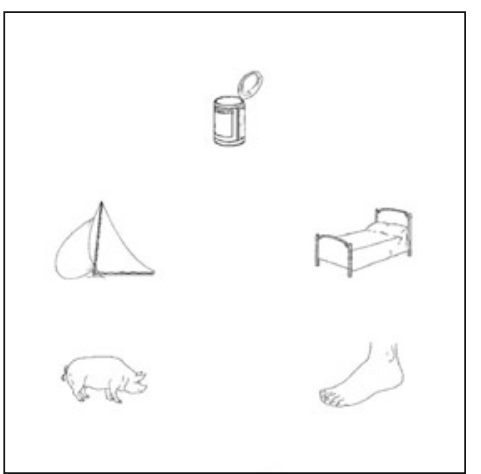

Array 1

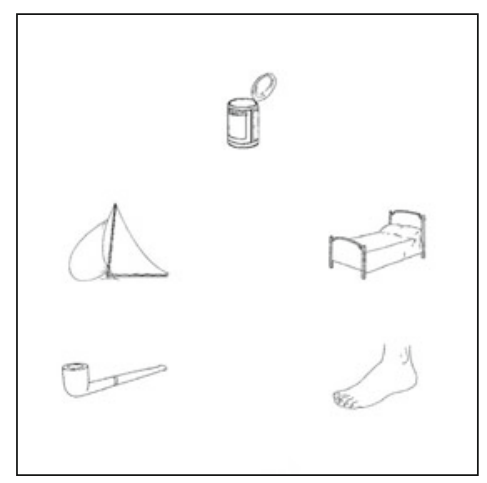

Array 2

Figure 1. Depiction of Arrays 1 and 2 in similar and dissimilar trials in Experiment 1 , in which two objects exchange identities. Item names on similar trials are phonologically similar, whereas those on dissimilar trials are not.

Change trials. For similar trials, 20 sets of six objects chosen on the basis of the phonological similarity of the initial phonemes of the object name were selected from the Snodgrass and Vanderwart (1980) corpus. Similar objects often shared their vowel phoneme as well. No objects assigned to a set had names that rhymed and no more than two were from the same basic-level semantic category. Each set of six objects was used to create two arrays of five-for example: Array 1, belt, bed, bee, bench, bear; Array 2, bell, bed, bee, bench, bear. The italicized items were the objects that changed between arrays. The initial phonemes of the items were all consonants, and all of the objects had monosyllabic names.

For dissimilar trials, the same 120 objects used in the phonologically similar arrays were recombined to create 20 phonologically dissimilar arrays in which the initial consonant and vowel phonemes of the names of the objects in the array were dissimilar. For example: Array 1, pig, can, sail, bed, foot; Array 2, pipe, can, sail, bed, foot. Note that the name of the substituted item (in italics) always shared the same initial phoneme and sometimes the vowel phoneme with the item replaced.

No-change trials. In no-change trials, Array 2 was identical to Array 1, so only five objects were displayed on each trial. A subset of 100 of the 120 objects used in change trials were recombined to create 20 similar no-change trials. These 100 objects were then recombined to create 20 dissimilar no-change trials.

Versions. Two sets of stimuli, Sets A and B, were created. The stimuli that served in change trials in Set A served as no-change stim- uli in Set B, and vice versa. Further, temporal order of array presentation was counterbalanced: Half the subjects saw Array 1 followed by Array 2; half saw the stimuli in the reverse order. This resulted in four versions of the task, with each object occurring twice in each version, once in a change trial and once in a no-change trial. Thus, each subject undertook 40 experimental trials, 10 in each of the four conditions (similar-change, dissimilar-change, similar-no change, and dissimilar-no change) in one of four versions of the stimuli. The four versions of the stimuli were also constructed so as to distribute the probability of change equally across all nine spatial positions of the arrays across trials. (Note that, within each version, object position remained unchanged between Arrays 1 and 2 in each trial.)

Stimulus properties. The names of objects in Array 1 and Array 2 were matched for mean: $n$ (letters), spoken frequency, written frequency, and neighborhood size using the CELEX (Baayen, Piepenbrock, \& van Rijn, 1993) and Kučera and Francis (1967) databases. The mean values for Arrays 1 and 2 in the similar and dissimilar change trials and for the similar and dissimilar no-change trials are shown in Table 1.

Items in similar and dissimilar arrays were selected on the basis of the phonology of their names rather than on the basis of their visual properties. Inspection of the arrays revealed little visual similarity between items in particular arrays, and attempts were made to minimize such similarity (given the other constraints on stimulus attributes) where it was present. There appeared to be no systematic differences between similar and dissimilar arrays in this respect. 
Table 1

Mean Values for Properties of the Names of Picture Stimuli Used in Experiments 1 and 2

\begin{tabular}{lrrrrrrrr}
\hline & \multicolumn{4}{c}{ Change Trials } & & \multicolumn{2}{c}{ No-Change Trials } \\
\cline { 2 - 4 } & $\begin{array}{c}\text { Similar } \\
\text { Array 1 }\end{array}$ & $\begin{array}{c}\text { Dissimilar } \\
\text { Array 2 }\end{array}$ & $\begin{array}{c}\text { Similar } \\
\text { Array 1 }\end{array}$ & $\begin{array}{c}\text { Dissimilar } \\
\text { Array 2 }\end{array}$ & & $\begin{array}{c}\text { Similar } \\
\text { Arrays 1 and 2 }\end{array}$ & $\begin{array}{c}\text { Dissimilar } \\
\text { Arrays 1 and 2 }\end{array}$ \\
\hline$N$ (letters) & 4 & 4 & 4 & 4 & & 4 & 4 \\
Spoken CELEX frequency & 177 & 183 & 176 & 187 & & 128 & 113 \\
Written CELEX frequency & 1,377 & 1,509 & 1,367 & 1,412 & & 1,488 & 1,349 \\
Written KF frequency & 79 & 87 & 75 & 85 & & 92 & 77 \\
Neighborhood size $(N)$ & 10 & 10 & 10 & 11 & & 10 & 10 \\
\hline
\end{tabular}

Note-CELEX spoken and written word frequencies can be found in Baayen, Piepenbrock, and van Rijn (1993) and KF written frequencies in Kučera and Francis (1967).

Procedure. Following their instructions, the subjects were given a 2-trial demonstration of the sequence of events in each trial, and then undertook 16 practice trials. The demonstration and practice trials included Snodgrass and Vanderwart (1980) pictures that were not used in the main experiment, and whose names were not from the same phonological groups used in the main task.

Each trial consisted of the following sequence of events: The word "Ready" appeared at the center of the screen for 1,000 msec; a fixation cross ("+") appeared for $500 \mathrm{msec}$; Array 1 appeared for 3,000 msec; a pattern mask appeared for 1,000 msec; Array 2 appeared until the subject responded or 2,000 msec had elapsed. At mask offset, a timer was started, and it was terminated by the response.

The subjects were required to press a key labeled $c$ (for change responses) or $n$ (for no-change responses) with their preferred hand. Response times (RTs) and accuracy were the dependent measures. Immediately following a subject's change-detection response, a reply box appeared on the screen. If a subject had indicated that he or she had not detected any change, the words "No Change" automatically appeared in the reply box, and the subject pressed the Return key of the keyboard to proceed to the next trial. If the subject had detected change, the word "Change" automatically appeared in the reply box. The subject was then required to type in the names of the objects that had changed and press the Return key to proceed to the next trial. The subjects were informed that across all trials, half the trials were change trials and half were not. They were instructed to respond as quickly and as accurately as possible. Presentation of trials was self-paced, and trial order was randomized for each subject individually.

\section{Results}

Mean percentage correct change detection scores are shown in Table 2 and mean percentage correct change identification scores are shown in Table 3. Correct change detection refers to the subject's accuracy in detecting that a change had occurred on a trial, without regard for accu-

Table 2

Mean Percentage of Accuracy and Mean Response Times (RTs, in Milliseconds) for Phonologically Similar and Dissimilar Change and No-Change Trials in Experiment 1

\begin{tabular}{llllll}
\hline & \multicolumn{2}{c}{$\%$ Correct } & & \multicolumn{2}{c}{ RT } \\
\cline { 2 - 3 } \cline { 5 - 6 } & $M$ & $S D$ & & $M$ & $S D$ \\
\hline Change Trials & & & & \\
$\quad$ Similar & 76.2 & 17.5 & & 1,750 & 413 \\
$\quad$ Dissimilar & 68.5 & 20.3 & & 1,727 & 459 \\
No-Change Trials & & & & \\
$\quad$ Similar & 96.2 & 7.0 & 1,488 & 424 \\
Dissimiliar & 96.2 & 5.7 & 1,506 & 559 \\
\hline
\end{tabular}

rate identification of the objects involved in the change. Change identification refers to the correct identification of at least one of the objects involved in the change, following change detection. This is typically the new object in Array 2, but it can be the object from Array 1 that is no longer present in Array 2, and subjects can of course report the changed items from both arrays.

A repeated measures ANOVA based on the percentage of correct change detection with the variables of trial type (change, no change) and phonological similarity (phonologically similar, dissimilar) yielded the following effects. Detection of no change $(96.2 \%)$ was substantially higher than detection of change $(72.3 \%)\left[F(1,25)=53.84, M S_{\mathrm{e}}=\right.$ $14,784.6, p<.0001]$. The main effect of phonological similarity was not significant, but the interaction of trial type and phonological similarity approached significance $\left[F(1,25)=3.75, M S_{\mathrm{e}}=384.6, p<.07\right]$. This result was entirely due to performance in the change condition. Change detection was not impaired by phonological similarity of object names - in fact, it was numerically more accurate for similar trials $(76.2 \%)$ than for phonologically dissimilar trials $(68.5 \%)$. For no-change trials, mean decision accuracy (also shown in Table 2) was high and did not differ for phonologically similar and dissimilar trials (96.2\%).

Performance on change and no-change trials was also used to calculate $d^{\prime}$ scores for the similar and dissimilar conditions (after replacement of 0 and 1.0 scores with .05 and .95 , respectively), and these are shown in Table 3. These scores provide detection estimates that are adjusted for bias; they did not differ significantly for similar and dissimilar trials. Table 3 also presents beta scores, which index response bias; these likewise did not differ significantly for similar and dissimilar trials.

Decision times were recorded; for the response latency analysis, errors and trials in which latencies were more than $2 S D$ s from the mean RT of all of a subject's trials (4\% of trials) were omitted from the analyses. Mean correct response latencies are shown in Table 2 for phonologically similar and dissimilar change and no-change trials. A two-way repeated measures ANOVA indicated a significant main effect of trial type, with faster responses to no-change than to change trials $\left[F(1,24)=9.62, M S_{\mathrm{e}}=\right.$ $243,077.9, p<.005]$. No other effects were significant. Thus, response latencies for phonologically similar and dissimilar change trials did not differ significantly, nor did 
Table 3

Mean $d^{\prime}$ and Beta Values for Phonologically Similar and Dissimilar Change Trials in Experiments 1 and 2

\begin{tabular}{|c|c|c|c|c|c|c|c|c|}
\hline & \multicolumn{4}{|c|}{ Experiment 1} & \multicolumn{4}{|c|}{ Experiment 2} \\
\hline & \multicolumn{2}{|c|}{ Similar } & \multicolumn{2}{|c|}{ Dissimilar } & \multicolumn{2}{|c|}{ Similar } & \multicolumn{2}{|c|}{ Dissimilar } \\
\hline & $M$ & $S D$ & $M$ & $S D$ & $M$ & $S D$ & $M$ & $S D$ \\
\hline$\overline{d^{\prime}}$ & 1.62 & 0.43 & 1.45 & 0.51 & 1.46 & 0.33 & 1.80 & 0.35 \\
\hline Beta & .81 & 0.39 & 0.69 & 0.58 & 1.34 & 0.44 & 1.26 & 0.55 \\
\hline
\end{tabular}

they differ significantly for the phonologically similar and dissimilar conditions of no-change trials.

Table 3 shows the percentages of trials in which the subject was able to accurately identify only the Array 1 changed object or only the Array 2 changed object or both the changed objects. The scores were subjected to a repeated measures ANOVA in which items identified (Array 1 only, Array 2 only, and both array items) and phonological similarity (similar, dissimilar) were the variables. There were significant differences in items identified $\left[F(2,50)=19.34, M S_{\mathrm{e}}=617.8, p<.0001\right]$. Contrast tests indicated that identification of the replaced item from the first array (4.2\%) was significantly less likely than identification of the new item in the second array $(25.8 \%)[F(1,50)=19.52, p<.0001]$, and that the identification rate for this second item did not differ significantly from joint identification of both the replaced and the new item $(33.5 \%)$. There was also a main effect of phonological similarity $\left[F(1,25)=4.50, M S_{\mathrm{e}}=68.9\right.$, $p<.05]$, with superior identification of changed objects on similar trials. Although the interaction was not significant, the means in Table 4 indicate that the phonological similarity effect was due to different levels of identification of the new item, when only one was reported. Identification of only the replaced item from the first array, or both of the items, $59.2 \%$, clearly exceeds the chance level of random selection of one item of five in Array 2, 20\%. The mean percentage incorrect report of an unchanged object (shown in Table 4) was considerably below $20 \%$ and did not differ significantly for the similar and dissimilar conditions.

\section{Discussion}

The results of Experiment 1 show that phonological similarity of object names in no way impaired changedetection object changes in simple arrays. This result was obtained with both hit rates and $d^{\prime}$ measures, and there was no indication that different levels of decision bias contributed, because beta values likewise showed no significant difference between conditions. Although the data showed that subjects were faster and more accurate at detecting that no change had taken place than detecting that a change had occurred, this decision was unaffected by the phonological similarity of the names of items in the array. Correct detection scores exceeded correct identification of one or both of the changed objects, a result previously reported in the flicker paradigm used by Rensink (2004). This observation suggests independent input from a visual STM system, such as the visuospatial sketchpad, which does not represent names of visual stimuli. As noted earlier, the visuospatial sketchpad and the phonological loop are assumed to be two independent slave systems of shortterm working memory providing input to the central executive. The finding that detection performance was not adversely affected by the phonological similarity of item names suggests that change detection need not require the phonological loop and verbal encoding, but may be based on input from the visuospatial sketchpad to the central executive. In contrast to Simons's (1996) concurrent verbal shadowing task, the task in Experiment 1 did not impose competing attentional demands of concurrent articulation on the resources available to the central executive to detect and identify change. Thus, the reduction in performance found in Simons's tasks may derive from a paucity of central executive resources available in dual-task performance. When identification of changed items was examined, phonological similarity was found to confer some advantage in this task.

Although there is evidence in the literature on STM recall that the phonological similarity effect can be abolished, the tasks showing this effect are different from the task of Experiment 1 (Nairne \& Kelley, 1999). Nairne and Kelley used up to $8 \mathrm{sec}$ of digit shadowing to abolish the effect, and up to $24 \mathrm{sec}$ of digit shadowing to show a reversal of the effect, and the dependent variable was reconstruction of correct serial order. The few list recall experiments that have examined effects of phonological similarity on item rather than serial recall have had mixed results; some have found null effects (Poirier \& SaintAubin, 1996; Watkins, Watkins, \& Crowder, 1974); some have found beneficial effects (Gathercole, Gardiner, \& Gregg, 1982; Wickelgren, 1965); and some have found adverse effects (Coltheart, 1993; Drewnowski, 1980). Fallon, Groves, and Tehan (1999) found a beneficial effect of phonological similarity when the items rhymed (instead of sharing similar phonemes), but detrimental effects of phonological similarity on accuracy of position in word lists (but see Fallon, Mak, Tehan, \& Daly, 2005).

The results of Experiment 1 are consistent with some models of short-term working memory (e.g., Nairne, 1988, 1990; Nairne \& Kelly, 1999; Tehan \& Fallon, 1999). These models support the idea that although within-list

Table 4

Mean Percentage of Identification of Changed Items in Phonologically Similar and Dissimilar Change Trials in Experiments 1 and 2

\begin{tabular}{|c|c|c|c|c|c|c|c|c|}
\hline & \multicolumn{4}{|c|}{ Experiment 1} & \multicolumn{4}{|c|}{ Experiment 2} \\
\hline & \multicolumn{2}{|c|}{ Similar } & \multicolumn{2}{|c|}{ Dissimilar } & \multicolumn{2}{|c|}{ Similar } & \multicolumn{2}{|c|}{ Dissimilar } \\
\hline & $M$ & $S D$ & $M$ & $S D$ & $M$ & $S D$ & $M$ & $S D$ \\
\hline Both & 33.5 & 22.4 & 33.5 & 24.3 & 39.8 & 18.2 & 47.1 & 22.0 \\
\hline Item $1^{*}$ & 3.8 & 5.7 & 4.6 & 7.1 & 20.8 & 12.1 & 22.9 & 12.9 \\
\hline Item 2 & 30.4 & 19.9 & 21.2 & 20.1 & - & & - & \\
\hline Wrong item & 8.5 & 9.2 & 9.2 & 11.6 & 2.9 & 4.6 & 4.6 & 6.6 \\
\hline
\end{tabular}

*Item 1 in Experiment 1 is the Array 1 object replaced in Array 2 by the object referred to as Item 2. In Experiment 2, Items 1 and 2 switched locations in Array 2, and both were present in Array 1, so report of either was included as Item 1 . 
phonological similarity may make it difficult to discriminate among items, there may be an overall beneficial effect of similarity because these items provide cues to facilitate retrieval of potentially eligible candidates for the list in question, in comparison with candidates from other lists, or nonlist items. For example, the phonologically similar Array 2 object names that begin with phoneme /b/ and share / $/$ / (belt, bed, bear, bench) may cue the subject to retrieve potential objects whose names share these phonemes, such as the correct Array 1 object, bell (in the Array 1 list bell, bed, bear, bench, bee), thus increasing the probability of a correct response. However, there was no evidence to suggest that the Array 1 object was more likely to be recalled for similar than for dissimilar arrays. Instead, the benefit of item similarity was due to correct identification of the new item in Array 2. We can only speculate that name similarity enhanced the familiarity judgment for old, unchanged items. Change detection, as noted earlier, was not always accompanied by identification of the changed objects, a finding also reported by Rensink (2004), and this phenomenon is reminiscent of the difference between "remember" and "know" responses in the list memory literature (e.g., Rajaram, 1993).

\section{EXPERIMENT 2}

Experiment 2 examined whether retention of spatial location information was adversely affected by the phonological similarity of the names of objects in arrays, using Simons's (1996) "switch" procedure: The same objects were shown in both arrays, and subjects were required to identify which of two objects in the array had exchanged places. Thus, subjects had to encode both object identity and spatial disposition and maintain this order-in-array information across the interstimulus interval, a task that might have similar demands to those of serial recall. Again, it was predicted that if phonological codes play some part in reporting changes and in identifying them, performance should be adversely affected by the phonological similarity of the names of objects in the arrays.

\section{Method}

Subjects. A new sample of 24 (17 female) Macquarie University undergraduates (mean age 18.5 years) participated for course credit. All reported normal or corrected-to-normal vision.

Design. The design was similar to that of Experiment 1 except that subjects were required to detect and identify the objects in the arrays that had exchanged places. Thus, object identities remained the same in Arrays 1 and 2, but the locations of two objects changed. Four versions of the arrays were constructed so as to distribute the probability of change as equally as possible across all nine spatial positions of the arrays across trials, and as in Experiment 1, to counterbalance temporal order of array. Each subject saw 80 trials: half change trials, half no-change trials. In half the trials, the names of the objects in the array were phonologically similar; in half, they were phonologically dissimilar. The subjects saw each object four times in each version, twice in a change trial and twice in a nochange trial. Because the same stimuli were present in both arrays, the properties of the names of the objects depicted (e.g., spoken frequency) were the same in both arrays.
Procedure. The procedure was identical to that of Experiment 1, except that when subjects indicated a change trial, they were required to report the names of the objects that exchanged locations.

\section{Results}

Mean percentages of correct change detection are shown in Table 5 and mean percentages of correct change identification are shown in Table 4. A two-way repeated measures ANOVA based on percentage of change-detection accuracy revealed a significant main effect of trial type, with detection on change trials $(69.1 \%)$ inferior to that on no-change trials $(96.8 \%)\left[F(1,23)=84.6, M S_{\mathrm{e}}=217.9\right.$, $p<.0001]$. Phonological similarity impaired detection $\left[F(1,23)=24.0, M S_{\mathrm{e}}=39.1, p<.0001\right]$, and there was a significant interaction between trial type and phonological similarity $\left[F(1,23)=12.2, M S_{\mathrm{e}}=45.1, p<.002\right]$, with planned contrasts indicating that the detrimental effect of phonological similarity was confined to change trials $[F(1,23)=32.5, p<.0001]$. Mean detection accuracy on no-change trials was high, and not appreciably different on similar and dissimilar trials.

Again, $d^{\prime}$ and beta values based on correct change detection and false alarms to unchanged arrays were calculated and are shown in Table 3. A statistical comparison of these scores showed that $d^{\prime}$ was significantly higher for the dissimilar than for the phonologically similar condition $[t(23)=4.96, S E=.07, p<.0001]$; beta values did not differ significantly between conditions. Thus, both accuracy and $d^{\prime}$ measures showed that subjects were less likely to detect that two objects had switched places when their names were phonologically similar than when the names were phonologically dissimilar, and that this difference was not due to differences in bias.

Table 4 shows the percentages of trials in which the subject was able to identify just one of the objects that switched places (but not both) and the percentages of trials on which both these objects were identified. For example, when bell and belt switched places, and the subject reported only that bell had a new location, or reported only that belt had moved, the trial was scored as a correct identification of one changed object. These scores were subjected to a repeated measures ANOVA, which yielded only significant main effects, as follows. There was a significant difference in the number of items identified $\left[F(1,23)=15.39, M S_{\mathrm{e}}=724.9, p<.001\right]$; subjects were more likely to report both switched objects $(43.4 \%)$ than

\section{Table 5}

Mean Percentage of Accuracy and Mean Response Times (RTs, in Milliseconds) for Phonologically Similar and Dissimilar Change and No-Change Trials in Experiment 2

\begin{tabular}{llllll}
\hline & \multicolumn{2}{c}{$\%$ Correct } & & \multicolumn{2}{c}{ RT } \\
\cline { 5 - 6 } & $M$ & $S D$ & & $M$ & $S D$ \\
\hline Change Trials & & & & \\
$\quad$ Similar & 63.5 & 14.3 & & 3,122 & 1,118 \\
$\quad$ Dissimilar & 74.6 & 16.2 & & 3,183 & 1,216 \\
No-Change Trials & & & & \\
$\quad$ Similar & 97.5 & 3.6 & 2,646 & 903 \\
$\quad$ Dissimiliar & 96.0 & 3.9 & 2,559 & 854 \\
\hline
\end{tabular}


to report only one $(21.9 \%)$. The effect of phonological similarity was significant $\left[F(1,23)=10.29, M S_{\mathrm{e}}=51.3\right.$, $p<.005]$, with fewer switched objects in phonologically similar arrays $(30.3 \%)$ reported than in dissimilar arrays $(35.0 \%)$. The attribution of change to the wrong object(s) was rare and did not differ significantly in the two conditions $(2.9 \%$ in the similar condition and $4.6 \%$ in the dissimilar condition). As in Experiment 1, identification of at least one of the two switched objects $(65.3 \%)$ considerably exceeded chance level (40\%).

Mean correct response latencies for each condition are shown in Table 5. Trials in which latencies were more than $2 S D$ s from the mean RT of all of a subject's trials (3.9\% of trials) were omitted from the latency analyses. An ANOVA of decision times indicated a significant effect of trial type (change, no change) $[F(1,23)=36.57$, $\left.M S_{\mathrm{e}}=198,312.5, p<.0001\right]$, with slower responses on change than on no-change trials. The main effect of phonological similarity was not significant, but its interaction with trial type was significant $[F(1,23)=4.80$, $\left.M S_{\mathrm{e}}=133,429.6, p<.05\right]$. This interaction was due to a marginal $(p<.09)$ effect of slower RT on similar in comparison with dissimilar no-change trials, along with the absence of any significant RT difference on similar and dissimilar change trials.

\section{Discussion}

The results of Experiment 2 for switch changes show that phonological similarity of object names impaired change detection. Again, this result was confirmed by an analysis of $d^{\prime}$ scores and was not due to differences in bias, beta. When a change had been detected in change trials, subjects correctly identified at least one and frequently both of the items that exchanged places on most of these trials. The pattern of results is consistent with previous research findings on immediate memory for word lists: Similar phonology results in reduced accuracy in report of serial order and, in Experiment 2, in memory for spatial locations.

In the switch task, observers are presented with exactly the same visual information about object identity in both arrays, and the names of objects in both arrays contain exactly the same phonological information. In contrast to the identity change objects in Experiment 1, objects with similar names in similar trials in Experiment 2 cannot offer subjects any cuing or predictive advantage over dissimilar trials, because phonology can only cue object names and not spatial position. Spatial information is assumed to be encoded by the visuospatial sketchpad, which is specialized for encoding this form of information. However, its accuracy in representing exact locations of spatially contiguous items may be limited, thus leading to reliance on phonological coding of order.

\section{GENERAL DISCUSSION}

The evidence from Experiments 1 and 2 is that phonological similarity of the names of drawn objects in simple five-item arrays disrupts memory encoding of spatial order but facilitates memory for the identity of objects. Observers in these experiments were unconstrained in the procedures they used to encode the arrays in memory, apart from the requirement to report changes detected. Given the results from the manipulation of phonology in a changedetection task, it appears - as it does in prior research findings, including those of Simons (1996) - that subjects use naming strategies to encode and remember the positions of items. One possible technique is to begin with the top leftmost object, name it, and then continue naming items from left to right, row by row. These ordered names can be compared with the objects in the second array, which can be inspected in the same left-to-right, top-to-bottom sequence. Of course, individuals are likely to differ in their selection of a specific starting item and in the direction of inspection and naming of other objects, and an individual's choices may differ between trials; it was therefore not possible to determine, with a limited number of trials, whether such strategies were regularly adopted.

One feature of the results of the reported experiments is the difference in accuracy in change and no-change trials. In change trials, observers in each of the experiments accurately detected that a change had taken place in about two thirds of the trials. In no-change trials, however, performance was consistently near ceiling. An explanation for these differences might be that no-change trials are less demanding on memory capacity. This may be the case in identity change trials. Successful detection of change in identity change trials requires the subject to hold in memory 5 items from Array 1, and compare them with 5 items in Array 2, a total memory load of 10 object tokens of six object types. In no-change trials, there are 10 tokens but only five object types. However, there are the same number of types and tokens present in change and no-change arrays in switch trials, yet no-change accuracy remained better than change-detection accuracy.

Another possibility is that observers initially scan the display for changes in the visual properties of the second array; such properties might include contour proximity and size differences between adjacent objects (Jiang, Chun, \& Olson, 2004; Jiang, Olson, \& Chun, 2000; Woodman, Vecera, \& Luck, 2003). They may then undertake a slower, serial process of item name checking that may be vulnerable to phonological similarity effects. Identity changes and switch changes do cause small changes to the global visual pattern of the arrays, and RTs in five-item arrays were significantly faster in no-change trials than in change trials, which may indicate that subjects spent less time on item matching or comparison processes in nochange trials. Evidence suggesting that visual feature comparisons occur comes from Hollingworth and Henderson's $(2002,2003)$ findings that subjects can frequently detect small changes in the orientation of objects in a scene, and though it may be possible for some general features of orientation to be verbally encoded - for example, "leftfacing chair"-it seems unlikely that specific orientation angles would be phonologically encoded. 
The two main findings from Experiments 1 and 2 are that phonological similarity of array items (1) did not impair change detection of a substituted object but facilitated identification of the changed object and (2) did disrupt detection and identification of location change. These findings are consistent with some models of short-term working memory (e.g., Nairne, 1988, 1990, 2002) that argue that item similarity reduces the predictive value of item features, so that items sharing similar phonology will not provide cues to unique items, thus leading to difficulties in retaining serial order. The data from the switch experiment in Experiment 2 support this view. Phonological similarity reduced accurate report of items that had changed locations, a serial order effect that has been found by others using very different experimental paradigms and stimuli (Nairne \& Kelly, 1999; Tehan \& Fallon, 1999). Further, in Experiment 1, phonological similarity facilitated identification of identity change.

In contrast, in the switch experiment, phonology had no predictive power with regard to location in either similar or dissimilar trials. Rather, the loss of distinctiveness of the items due to phonological similarity led to less accurate recall of the sequence of objects from the first array and impaired accuracy of the comparison process used to detect location changes. These results indicate that phonological codes and their maintenance contribute to performance in change detection and that difficulties in establishing and maintaining these codes result in change blindness.

Real-world scenes and photographs of them contain more context, objects, and far more detail than do the stimuli used in Experiments 1 and 2. However, in real-world settings, people are not expected to attend to all the available information and do not expect to have to report changes that might occur. In fact, in Grimes's (1996) study — which did not explicitly demand attention to and report of details in scenes, but required change detection-many remarkably large alterations between successive fixations went unnoticed. For example, $50 \%$ failed to notice that two cowboys sitting on a bench exchanged heads. Our results suggest that focused attention and possibly explicit verbal encoding of scene details are required to ensure reliable change detection and identification. Thus, if the face of the cowboy on the left attracted explicit attention and verbal encoding (e.g., "he looks like my friend Joe"), the head change would be more likely to be detected.

The stimuli used in these experiments were line drawings. It is likely that, when more naturalistic stimuli are used (such as photographs of more complex natural scenes, in which objects vary in size, color, pattern, and other stimulus properties), verbal encoding may play an even greater role as memory load increases. The evidence presented here is that phonological similarity of the names of objects in scenes may have a positive effect on detection of identity changes in scenes but will have a detrimental effect on the extent to which the locations of those change items are accurately recalled. Simons (1996) showed that concurrent articulation reduced recall of such changes to chance. In the absence of concurrent articulation, the evidence here is that object location may be more difficult to specify accurately if the names of the objects concerned share phonology.

The results also indicate that visual STM, a system exemplified by the visuospatial sketchpad, is used to encode and maintain information between successive views of an array or scene. The rapid, accurate decisions of no change across views of arrays of objects with similar and dissimilar names are most likely to be affected by this visual memory system. It can provide input that the stored and current objects differ in some way in their features or orientation; it can also, possibly preattentively, detect large gaps in a previously occupied spatial area, or the filling of prior empty regions. However, as we have noted, detection of smaller spatial location changes benefits from explicit phonological coding of the order of objects in the array. Experiments 1 and 2 demonstrate concurrent deployment of visuospatial and phonological working memory in the processes of change detection.

\section{REFERENCES}

Avons, S. E., \& Phillips, W. A. (1987). Representation of matrix patterns in long- and short-term visual memory. Acta Psychologica, 65, 227-246.

BAAYen, R. H., Piepenbrock, R., \& van Rijn, H. (1993). The CELEX lexical database (CD-ROM). Philadelphia: Linguistic Data Consortium, University of Pennsylvania.

BADDELEY, A. D. (1966). Short-term memory for word sequences as a function of acoustic, semantic and formal similarity. Quarterly Journal of Experimental Psychology, 18, 362-365.

BADDELEY, A. D. (1968). How does acoustic similarity influence shortterm memory? Quarterly Journal of Experimental Psychology, 20, 249-264.

BADDELEY, A. [D.] (1986). Working memory. Oxford: Oxford University Press.

Bates, T. C., \& D’Oliveiro, L. (2003). PsyScript: A Macintosh application for scripting experiments. Behavior Research Methods, Instruments, \& Computers, 35, 565-576.

COLTHEART, V. (1993). Effects of phonological similarity and concurrent irrelevant articulation on short-term memory recall of repeated and novel word lists. Memory \& Cognition, 21, 539-545.

Coltheart, V. (1999). Comparing short-term memory and memory for rapidly presented visual stimuli. International Journal of Psychology, 34, 293-300.

CoNRAD, R. (1963). Acoustic confusions and memory span for words. Nature, 197, 1029-1030.

CoNRAD, R. (1964). Acoustic confusions in immediate memory. British Journal of Psychology, 55, 75-84.

ConRad, R. (1971). The chronology of the development of covert speech in children. Developmental Psychology, 5, 398-405.

Conrad, R. (1972). The developmental role of vocalizing in short-term memory. Journal of Verbal Learning \& Verbal Behavior, 11, 521533.

CONRAD, R., \& HULL, A. J. (1964). Information, acoustic confusion, and memory span. British Journal of Psychology, 55, 429-432.

DREWNOWSKI, A. (1980). Attributes and priorities in short-term recall: A new model of memory span. Journal of Experimental Psychology: General, 109, 208-250.

Fatlon, A. B., Groves, K., \& Tehan, G. (1999). Phonological similarity and trace degradation in the serial recall task: When CAT helps RAT, but not MAN. International Journal of Psychology, 34, 301307.

FALLON, A. B., MaK, E., Tehan, G., \& Daly, C. (2005). Lexicality and phonological similarity: A challenge for the retrieval-based account of serial recall? Memory, 13, 349-356.

Gathercole, S. E., Gardiner, J. M., \& GregG, V. H. (1982). Modality 
and phonological similarity effects in serial recall: Does one's own voice play a role? Memory \& Cognition, 10, 176-180

Grimes, J. (1996). On the failure to detect changes in scenes across saccades. In K. A. Akins (Ed.), Perception (Vancouver Studies in Cognitive Science, Vol. 5, pp. 89-110). New York: Oxford University Press.

Halliday, M. S., Hitch, G. J., Lennon, B., \& Pettipher, C. (1990). Verbal short-term memory in children: The role of the articulatory loop. European Journal of Cognitive Psychology, 2, 23-38.

Hintzman, D. L. (1965). Classification and aural coding in short-term memory. Psychonomic Science, 3, 161-162.

Hintzman, D. L. (1967). Articulatory coding in short-term memory. Journal of Verbal Learning \& Verbal Behavior, 6, 312-316.

Hitch, G. J., \& HALlidaY, M. S. (1983). Working memory in children. Philosophical Transactions of the Royal Society of London: Series B, 302, 325-340.

HiTch, G. J., Woodin, M. E., \& BAKer, S. (1989). Visual and phonological components of working memory in children. Memory \& Cognition, 17, 175-185

Hollingworth, A., \& Henderson, J. M. (2002). Accurate visual memory for previously attended objects in natural scenes. Journal of Experimental Psychology: Human Perception \& Performance, 28, 113-136.

HollingWorth, A., \& Henderson, J. M. (2003). Testing a conceptual locus for the inconsistent object change detection advantage in realworld scenes. Memory \& Cognition, 31, 930-940.

Jiang, Y., Chun, M. M., \& Olson, I. R. (2004). Perceptual grouping in change detection. Perception \& Psychophysics, 66, 446-453.

Jiang, Y., Olson, I. R., \& CHUN, M. M. (2000). Organization of visual short-term memory. Journal of Experimental Psychology: Learning, Memory, \& Cognition, 26, 683-702.

KUČERA, H., \& FRANCIS, W. N. (1967). Computational analysis of presentday American English. Providence, RI: Brown University Press.

Levin, D. T., \& Simons, D. J. (1997). Failure to detect changes to attended objects in motion pictures. Psychonomic Bulletin \& Review, 4, 501-506.

LONGONI, A. M., Richardson, J. T., \& Aiello, A. (1993). Articulatory rehearsal and phonological storage in working memory. Memory \& Cognition, 21, 11-22.

LoNGONI, A. M., \& ScAlisi, T. G. (1994). Developmental aspects of phonemic and visual similarity effects: Further evidence in Italian children. In A. de Ribaupierre \& G. J. Hitch (Eds.), Development of working memory (pp. 57-71). Hove, U.K.: Erlbaum.

MacAndrew, D. K., Klatzky, R. L., Fiez, J. A., McClelland, J. L., \& BECKER, J. T. (2002). The phonological-similarity effect differentiates between two working memory tasks. Psychological Science, 13, 465-468.

MCCONKIE, G. W., \& CURRIE, C. B. (1996). Visual stability across saccades while viewing complex pictures. Journal of Experimental Psychology: Human Perception \& Performance, 22, 563-581.

NAIRNE, J. S. (1988). A framework for interpreting recency effects in immediate serial recall. Memory \& Cognition, 16, 343-352.

NAIRne, J. S. (1990). A feature model of immediate memory. Memory \& Cognition, 18, 251-269.

NAIRNE, J. S. (2002). Remembering over the short-term: The case against the standard model. Annual Review of Psychology, 53, 53-81.
Nairne, J. S., \& Kelley, M. R. (1999). Reversing the phonological similarity effect. Memory \& Cognition, 27, 45-53.

Noizet, G., \& PyNTE, J. (1976). Implicit labelling and readiness for pronunciation during the perceptual process. Perception, 5, 217-223.

O'Regan, J. K., RensinK, R. A., \& Clark, J. J. (1996). "Mud splashes" render picture changes invisible. Investigative Ophthalmology \& $\mathrm{Vi}$ sual Science, 37, S213.

PoIrIer, M., \& SAINT-Aubin, J. (1996). Immediate serial recall, word frequency, item identity and item position. Canadian Journal of Experimental Psychology, 50, 408-412.

PoTTER, M. C., \& FAUlCONER, B. A. (1975). Time to understand pictures and words. Nature, 253, 437-438.

RAJARAM, S. (1993). Remembering and knowing: Two means of access to the personal past. Memory \& Cognition, 21, 89-102.

Rensink, R. A. (2004). Visual sensing without seeing. Psychological Science, 15, 27-32

Rensink, R. A., O'Regan, J. K., \& Clark, J. J. (1995). Global transients disrupt visual perception of changes in scenes (Tech. Rep. No. 95-6). Cambridge, MA: Cambridge Basic Research.

Schiano, D. J., \& WatKins, M. J. (1981). Speech-like coding of pictures in short-term memory. Memory \& Cognition, 9, 110-114

Schweickert, R., Guentert, L., \& Hersberger, L. (1990). Phonological similarity, pronunciation rate, and memory span. Psychological Science, 1, 74-77.

SiMONS, D. J. (1996). In sight, out of mind: When object representations fail. Psychological Science, 7, 301-305.

Simons, D. J., \& LEVIN, D. T. (1998). Failure to detect changes to people during a real-world interaction. Psychonomic Bulletin \& Review, 5, 644-649.

SimONS, D. J., \& Rensink, R. A. (2005). Change blindness: Past, present, and future. Trends in Cognitive Sciences, 9, 16-20.

SNODGRASS, J. G., \& VANDERWART, M. (1980). A standardized set of 260 pictures: Norms for name agreement, image agreement, familiarity, and visual complexity. Journal of Experimental Psychology: Human Learning \& Memory, 6, 174-215.

Te HAN, G., \& FALlON, A. B. (1999). A connectionist model of shortterm cued recall. In J. Wiles \& T. Dartnall (Eds.), Perspectives on cognitive science: Vol. 2. Theories, experiments, and foundations (pp. 221-237). Stamford, CT: Ablex.

WALLIS, G., \& BüLthoFF, H. (2000). What's scene and not seen: Influences of movement and task upon what we see. Visual Cognition, 7, 175-190

WATKINS, M. J., WATKINS, O. C., \& Crowder, R. G. (1974). The modality effect in free and serial recall as a function of phonological similarity. Journal of Verbal Learning \& Verbal Behavior, 13, 430-447.

WICKELGREN, W. A. (1965). Short-term memory for phonemically similar lists. American Journal of Psychology, 78, 567-574.

Woodman, G. F., Vecera, S. P., \& LuCK, S. J. (2003). Perceptual organization influences visual working memory. Psychonomic Bulletin \& Review, 10, 80-87.

ZELINSKY, G. J., \& MurPhy, G. L. (2000). Synchronizing visual and language processing: An effect of object name length on eye movements. Psychological Science, 11, 125-131.

(Manuscript received July 19, 2004; revision accepted for publication October 21, 2005.) 